\section{A Passive Repetitive Controller for Discrete-Time Finite-Frequency Positive-Real Systems}

Ramon Costa-Castelló, Danwei Wang, and Robert Griñó

\begin{abstract}
This work proposes and studies a new internal model for discrete-time passive or finite-frequency positive-real systems which can be used in repetitive control designs to track or to attenuate periodic signals. The main characteristic of the proposed internal model is its passivity. This property implies closed-loop stability when it is used with discrete-time passive plants, as well as the broader class of discrete-time finite-frequency positive real plants. This work discusses the internal model energy function and its frequency response. A design procedure for repetitive controllers based on the proposed internal model is also presented. Two numerical examples are included.
\end{abstract}

Index Terms-Discrete-time control, discrete-time passivity, finite-frequency positive realness, repetitive control.

\section{INTRODUCTION}

Repetitive control is an established control design technique for systems handling periodical signals. The most important component in a repetitive controller is the periodic signal internal model [4]. The main drawback of the conventional internal model is its high order that makes the stability analysis of the closed-loop system difficult. In the pioneering work of Inoue [10], the stability of these systems is established by disecting the closed-loop system into three series-connected subsystems. The stability checking of the first two subsystems is straightforward but, for the remaining third subsystem, the Small Gain Theorem needs to be used. Later, this approach was extended and modified to improve high frequency robustness [7] and $H^{\infty}$ conditions and procedures were established [19]. Lyapunov based analysis was also introduced in [14]. These works provide stability conditions for passive (i.e. Positive Real (PR) in linear systems) systems. These results have been extended to Almost Strictly Positive Real (ASPR) and Almost Strictly Negative Real (ASNR) systems [3]. Although a discrete-time formulation exists [1], most works have set out and developed repetitive control in continuous time.

This work proposes a new structure for the internal model which is discrete-time passive (equivalently, it is Discrete-Time Positive Real). When this internal model is used as a repetitive controller with a discrete-time passive plant, the closed-loop system stability is guaranteed. Furthermore, feedback passivizable plants [16] can also benefit from this property.

The passivity property of the proposed internal model implies a reduced phase lag and this finds its application to finite frequency positive-real (FFPR) and passive plants [11]. The FFPR property is less

Manuscript received October 02, 2006; revised June 29, 2007. First published March 24, 2009; current version published April 08, 2009. This work was supported in part by the Comision Interministerial de Ciencia y Tecnología (CICYT) under Project DPI2007-62582. Recommended by Associate Editor M. Kothare.

R. Costa-Castelló and R. Griñó are with the Institut d'Organització i Control de Sistemes Industrials (IOC), Universitat Politècnica de Catalunya (UPC), Barcelona 08028, Spain (e-mail: ramon.costa-castello@ieee.org; roberto.grino@upc.edu).

D. Wang is with the School of Electrical and Electronics Engineering, Nanyang Technological University (NTU), Singapore 639798 (e-mail: edwwang@ntu.edu.sg).

Color versions of one or more of the figures in this technical note are available online at http://ieeexplore.ieee.org.

Digital Object Identifier 10.1109/TAC.2008.2009594 restrictive than passivity. In addition, being positive real in a certain frequency range is a necessary condition for good control performance in that frequency range [12].

The proposed internal model introduces configurable zeros to shape the open-loop frequency response and to provide an additional degree of freedom in control design. In view of time response, the proposed internal model will reduce the time delay of repetitive controllers due to its reduced phase lag. Furthermore, several internal models can be combined in parallel to obtain a multiperiodic repetitive control system. Besides this, the proposed internal model preserves all relevant properties of repetitive controllers: trajectory tracking and disturbance rejection capability, simple structure and low computational cost.

This work analyses and characterises the proposed internal model and presents a design methodology for FFPR discrete-time plants which is illustrated with two numerical examples.

\section{PASSIVE INTERNAL MODEL}

\section{A. Internal Model Structure}

The proposed internal model is described by the transfer function

$$
K(z)=\frac{Y(z)}{U(z)}=k_{r} \frac{z^{N}-H(z) \beta}{z^{N}-H(z) \alpha}
$$

where $N \in \mathbb{N}, k_{r} \in \mathbb{R}^{+},-1 \leq \alpha \leq 1,-1 \leq \beta \leq 1$ and $H(z)$ is a low-pass filter.

For $H(z)=1$, the poles of (1) are ${ }^{1}$ $p_{k}=\sqrt[N]{|\alpha|} e^{j((2 \pi / N) k+(\pi(1-\operatorname{sign}(\alpha)) / 2 N))}, k=0,1, \ldots, N-1$, so they are uniformly distributed over a circumference of radius $^{2} \sqrt[N]{\alpha}$. The frequencies associated to the poles are $\omega_{k}=(2 \pi / N) k+(\pi(1-\operatorname{sign}(\alpha)) / 2 N)$, so the poles are placed to cover all the harmonic frequencies of the fundamental one, $2 \pi / N$. This pole placement is the same as the one obtained in the conventional internal model. The zeroes of (1) follow a similar placement [2].

Depending on the signs of $\alpha$ and $\beta$, we have the following cases: if $\operatorname{sign}(\beta)=\operatorname{sign}(\alpha)$, the poles and the zeroes are placed at the same frequencies, and if $\operatorname{sign}(\beta) \neq \operatorname{sign}(\alpha)$ the poles and the zeroes are placed at shifted frequencies. In particular, in the later case, the frequencies associated with the zeros are exactly the mean of the frequencies of the adjacent poles. However, in real world applications it is necessary to reduce the controller gain in the high frequency band and for this reason, the internal model includes the low-pass filter $H(z)$.

\section{B. Energy Properties of the Passive Internal Model}

Let $(\mathbf{A}, \mathbf{B}, \mathbf{C}, \mathbf{D})$ be the LTI discrete-time system

$$
\begin{aligned}
\mathbf{x}_{k+1} & =\mathbf{A} \mathbf{x}_{k}+\mathbf{B} \mathbf{u}_{k} \\
\mathbf{y}_{k} & =\mathbf{C} \mathbf{x}_{k}+\mathbf{D} \mathbf{u}_{k}
\end{aligned}
$$

where $\mathbf{x}_{k} \in \mathbb{R}^{n}, \mathbf{y}_{k}, \mathbf{u}_{k} \in \mathbb{R}^{m}$; or in input-output form ${ }^{3} \mathbf{G}(z) \triangleq$ $\mathbf{C}\left(z \mathbf{I}_{n}-\mathbf{A}\right)^{-1} \mathbf{B}+\mathbf{D}$.

Definition 1 (Discrete-Time Passivity (DTP), [13]): System (2), (3) is discrete-time passive with storage function $V_{k}=(1 / 2) x_{k}^{T} \mathbf{P} x_{k}$ ( $V$-passive) if, and only if

$$
\Delta V_{k} \triangleq V_{k+1}-V_{k} \leq \mathbf{y}_{k}^{T} \mathbf{u}_{k} .
$$

Definition 2 (( $\mathbf{Q}, \mathbf{S}, \mathbf{R})$-Dissipative [5]): DTP systems with

$$
\Delta V_{k}=\mathbf{y}_{k}^{T} \mathbf{Q} \mathbf{y}_{k}+2 \mathbf{y}_{k}^{T} \mathbf{S} \mathbf{u}_{k}+\mathbf{u}_{k}^{T} \mathbf{R} \mathbf{u}_{k}
$$

$1_{\operatorname{sign}}(x)$ equals 1 for $x \geq 0$ and -1 for $x<0$.

${ }^{2}|\alpha|<1$ is necessary to assure the stability of the repetitive block.

${ }^{3} \mathbf{I}_{n}$ being the $n$th order identity matrix. 
where $\mathbf{Q}$ and $\mathbf{R}$ are symmetric matrices and $\mathbf{S}$ an appropriate size matrix, are regarded as $(\mathbf{Q}, \mathbf{S}, \mathbf{R})$-Dissipative systems.

Lemma 1 ([5]): If a single-input-single-output (SISO) system $(\mathbf{A}, \mathbf{B}, \mathbf{C}, \mathbf{D})$ with transfer function $G(z)$ is $(Q, S, R)-D T P$, then:

1) If $Q<0$, then the graph of $G\left(e^{j \omega}\right)$ lies inside the circle on the complex plane with center $S /|Q|$ and radius $(1 /|Q|) \sqrt{S^{2}+R|Q|}$.

2) If $Q=0$, then the graph of $G\left(e^{j \omega}\right)$ lies to the right (if $S>0$ )or the left (if $S<0$ ) of the vertical line $\operatorname{Re}\{z\}=-(R / 2 S)$.

For $H(z)=1$, a state-space description of the transfer function in (1) has the matrices ${ }^{4} \mathbf{A}=\left[\begin{array}{cc}\mathbf{0}_{n-1} & I_{n-1} \\ \alpha & \mathbf{0}_{n-1}^{T}\end{array}\right], \mathbf{B}=\left[\begin{array}{c}\mathbf{0}_{n-1} \\ 1\end{array}\right], \mathbf{C}=$ $\left[k_{r}(\alpha-\beta), \mathbf{0}_{n-1}^{T}\right]$ and $\mathbf{D}=\left[k_{r}\right]$

Proposition 1: The passive internal model in (1), for $H(z)=1$ and $k_{r}>0,|\alpha| \leq 1,|\beta| \leq 1$ and $\alpha \beta \neq 1$, is $(Q, S, R)$-Dissipative and also DTP.

Proof: Through inspection, $\Delta V_{k}$ can be written as in (5) by using $\mathbf{P}=\left(k_{r}(\beta-\alpha)^{2} /(1-\beta \alpha)\right) \mathbf{I}_{n}, Q=\left(1-\alpha^{2}\right) / 2 k_{r}(\beta \alpha-1), R=$ $k_{r}\left(1-\beta^{2}\right) / 2(\beta \alpha-1)$ and $S=1 / 2$. Also, according to Definition 4 and noting that $S=1 / 2, Q<0$ and $R<0$ it is clear that the system is DTP.

Definition 3 (Discrete-Time Positive Real (DTPR)): Let $\mathbf{G}(z)$ be a square matrix of real rational functions. Then $\mathbf{G}(z)$ is called DiscreteTime Positive Real (DTPR) if it satisfies the following properties:

a) The entries of $\mathbf{G}(z)$ are analytic in $|z|>1$.

b) Every pole of $\mathbf{G}(z)$ on $z=e^{j \omega}$, if any, is simple and the corresponding residue matrix is Hermitian positive semidefinite.

c) $\mathbf{G}\left(e^{j \omega}\right)+\mathbf{G}^{*}\left(e^{j \omega}\right) \geq 0, \forall|\omega| \leq \pi$.

Remark 1: It is important to remark that in linear systems DTPR is equivalent to DTP. The connection between both definitions is provided by the discrete-time KYP-Lemma [9].

Proposition 2: If $k_{r}>0,|\alpha| \leq 1,|\beta| \leq 1, \alpha \beta \neq 1$ with $H(z)$ stable, $H(1)=1$ and $\left|H\left(e^{j \omega}\right)\right|<1,0<|\omega|<\pi$ the internal model, $K(z)=k_{r}\left(\left(z^{N}-\beta H(z)\right) /\left(z^{N}-\alpha H(z)\right)\right)$, introduced in (1), is DTPR.

Proof: The proof is organized in the following way: firstly, we show that the Nyquist plot of $K^{\prime}(z)=k_{r}\left(\left(z^{N}-\beta\right) /\left(z^{N}-\alpha\right)\right)$ decomposes the complex plane into two connected regions; secondly, we show that the curves $k_{r}\left(\left(e^{j \omega N}-\beta\right) /\left(e^{j \omega N}-\alpha\right)\right)$ and $k_{r}\left(\left(e^{j \omega N}-\beta H\left(e^{j \omega}\right)\right) /\left(e^{j \omega N}-\alpha H\left(e^{j \omega}\right)\right)\right)$ have only one point in common; thirdly, we show that another point is in one of the partitions defined by the Nyquist plot of $K^{\prime}(z)$ such that the curve is DTPR. Finally, it is proved that $K(z)$ is stable. This procedure, together with the assumption that the Nyquist plot of $K(z)$ is continuous, will prove that it is DTPR.

1) The Nyquist plot $K^{\prime}(z)$ defines a complex plane partition. In Proposition 1, it has been stated that $K^{\prime}(z)$ is DTP, so it is DTPR; or equivalently, the Nyquist plot of $K^{\prime}(z)$ lies in the open right half complex plane. The topology will be analyzed in two different cases:

- In case $|\alpha|<1$, the Nyquist plot of $K(z)$ is a circumference of radius $r=k_{r}\left(|\alpha-\beta| /\left(1-\alpha^{2}\right)\right)$ and center $c=k_{r}((1-$ $\left.\beta \alpha) /\left(1-\alpha^{2}\right)\right)$. In this case, the two partitions are the interior and exterior of this circumference.

- In case $|\alpha|=1, \operatorname{Re}\left\{K\left(e^{j \omega}\right)\right\}=(1+\beta) / 2$. In this case the two partitions are the left and right half plane of the vertical line $z=(1+\beta) / 2$.

2) The Nyquist plots of $K(z)$ and $K^{\prime}(z)$ have only one point in common which is the initial point $z=1(\omega=0)$. To prove this statement lets assume that another intersection exists, so $k_{r}\left(\left(e^{j \omega_{1} N}-\beta\right) /\left(e^{j \omega_{1} N}-\alpha\right)\right)=$

${ }^{4} \mathbf{O}_{n}$ being the $n$th order zero column vector. $k_{r}\left(\left(e^{j \omega_{2} N}-\beta H\left(e^{j \omega_{2}}\right)\right) /\left(e^{j \omega_{2} N}-\alpha H\left(e^{j \omega_{2}}\right)\right)\right)$ should be satisfied; which implies $e^{j \omega_{2} N}=e^{j \omega_{1} N} H\left(e^{j \omega_{2}}\right)$. The only possible solution is for $\left|H\left(e^{j \omega_{2}}\right)\right|=1$ and, according to the hypothesis made on $H$, this is only feasible for $\omega_{2}=0$. Note that if $\alpha=1$ this point is placed at $\infty$.

3) $K(-1)=k_{r}\left(\left((-1)^{N}-\beta H(-1)\right) /\left((-1)^{N}-\alpha H(-1)\right)\right) \in \mathbb{R}$ is inside one of the partitions.

- In the case of $|\alpha|<1$ it is sufficient to prove that it is inside the circle described by $K^{\prime}\left(e^{j \omega}\right)$. In order to fulfill this, it is necessary that $\left(\left(\left((-1)^{N}-\beta H(-1)\right) /\left((-1)^{N}-\alpha H(-1)\right)\right)-((1-\right.$ $\left.\left.\alpha \beta) /\left(1-\alpha^{2}\right)\right)\right)^{2} \leq(\alpha-\beta)^{2} /\left(1-\alpha^{2}\right)^{2}$ which can be rewritten as $-(\alpha-\beta)^{2}\left(1-H(-1)^{2}\right)<0$. This inequality is always true in the proposition conditions $(H(-1)<1)$.

- In the case $|\alpha|=1$. It is not difficult to prove that $k_{r}\left(\left((-1)^{N}-\right.\right.$ $\left.\beta H(-1)) /\left((-1)^{N}-\alpha H(-1)\right)\right)-((1+\beta) / 2) k_{r}>0$. So the Nyquist plot of $K(z)$ lies in the right half plane of the Nyquist plane.

4) $K(z)$ is analytic in $|z|>1$. By writing $K(z)$ as a diagram and applying the Small Gain Theorem it can be shown that the system is stable (marginally stable for $|\alpha|=1$ ).

It has been proven that the Nyquist plot of $K(z)$ lies in the right half plane of the Nyquist plane and that it is analytic for $|z|>1$, so $K(z)$ is DTPR and, then, DTP.

Remark 2: The parallel connection of two or more passive systems yields a passive system. So it is possible to use several internal models, with different values of $N$, and its parallel connection will be passive. This property can be used in order to design discrete-time multi-periodic repetitive controllers [15].

\section{Passive Internal Model Frequency Response}

Proposition 3: If $k_{r}>0,|\alpha| \leq 1,|\beta| \leq 1$ and $H(z)$ stable with $\left|H\left(e^{j \omega}\right)\right|<h \forall \omega \in[\varpi, \pi]$, then the Nyquist plot of $K(z)=$ $k_{r}\left(\left(z^{N}-\beta H(z)\right) /\left(z^{N}-\alpha H(z)\right)\right)$ lies in a disk of center $c=k_{r}((1-$ $\left.\left.\beta \alpha h^{2}\right) /\left(1-\alpha^{2} h^{2}\right)\right)+j 0$ and radius $r=k_{r}\left(h|\alpha-\beta| /\left(1-\alpha^{2} h^{2}\right)\right)$.

Proof: The proof follows the same steps as those in Proposition 2. By applying Lemma 1 and Proposition 1 to $k_{r}\left(\left(z^{N}-\beta h\right) /\left(z^{N}-\alpha h\right)\right)$, it can be shown that its Nyquist plot is a circumference of radius $r=$ $k_{r}\left(h|\alpha-\beta| /\left(1-\alpha^{2} h^{2}\right)\right)$ and center $c=k_{r}\left(\left(1-\beta \alpha h^{2}\right) /\left(1-\alpha^{2} h^{2}\right)\right)+$ $j 0$.

In order to prove that $K(z)$ lies in the abovementioned circle it must be proved that if $\left|H\left(e^{j \omega}\right)\right|<h$ no intersection between $k_{r}\left(\left(e^{j \omega_{1} N}-\beta h\right) /\left(e^{j \omega_{1} N}-\alpha h\right)\right)$ and $k_{r}\left(\left(e^{j \omega_{2} N}-\right.\right.$ $\left.\left.\beta H\left(e^{j \omega_{2}}\right)\right) /\left(e^{j \omega_{2} N}-\alpha H\left(e^{j \omega_{2}}\right)\right)\right)$ exists and that the last point $\left(\omega_{2}=\pi\right)$, of the second curve, is inside the circle.

1) An intersection between the circumference and the Nyquist plot of $K(z)$ in $\omega \in[\varpi, \pi]$ should satisfy: $\left(\left(e^{j \omega_{1} N}-\beta h\right) /\left(e^{j \omega_{1} N}-\right.\right.$ $\alpha h))=\left(\left(e^{j \omega_{2} N}-\beta H\left(e^{j \omega_{2}}\right)\right) /\left(e^{j \omega_{2} N}-\alpha H\left(e^{j \omega_{2}}\right)\right)\right)$ which is equivalent to: $h e^{j \omega_{2} N}=H\left(e^{j \omega_{2}}\right) e^{j \omega_{1} N}$. Note that this equation has no solution in $\omega_{2} \in[\varpi, \pi]$ due to the fact that $\left|H\left(e^{j \omega}\right)\right|<h$ $\forall \omega \in[\varpi, \pi]$. So, no intersection between the circumference and the Nyquist plot of $K(z)$ in the frequency range $[\varpi, \pi]$ exists.

2) The last point of the Nyquist plot of $K(z)(z=-1)$ is in the circle defined by the Nyquist plot of $k_{r}\left(\left(z^{N}-\right.\right.$ $\left.\beta h) /\left(z^{N}-\alpha h\right)\right)$. This fact can be proved by showing that: $\left(\left(\left((-1)^{N}-\beta H(-1)\right) /\left((-1)^{N}-\alpha H(-1)\right)\right)-((1-\right.$ $\left.\left.\left.\alpha \beta h^{2}\right) /\left(1-\alpha^{2} h^{2}\right)\right)\right)^{2} \leq\left(h|\alpha-\beta| /\left(1-\alpha^{2} h^{2}\right)\right)^{2}$ that is equivalent to $\left|H(-1)+\alpha h^{2}\right| \leq h|1+\alpha H(-1)|$ which can be easily proven true by assuming $|\alpha|<1$ and $|H(-1)|<h<1$.

As the Nyquist plot of $K(z)$ in $\omega \in[\varpi, \pi]$ is smooth, does not cross the circumference and has one point inside, the complete curve is in the circle.

Corollary 1: The gain of the internal model, $\left|K\left(e^{j \omega}\right)\right|$, in the frequency range $[\varpi, \pi]$, for $k_{r}>0,|\alpha| \leq 1,|\beta| \leq 1, H(z)$ stable and 


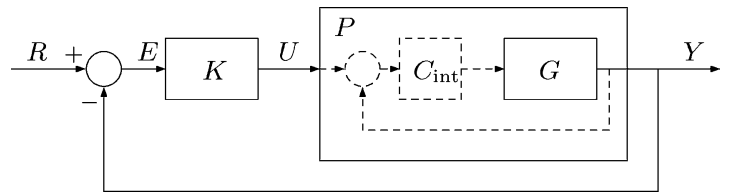

Fig. 1. Closed-loop controlled system block diagram.

$\left|H\left(e^{j \omega}\right)\right|<h, \forall \omega \in[\varpi, \pi]$ is in the interval $\left[\min \left\{k_{r}((1-h \beta) /(1-\right.\right.$ $\left.h \alpha)), k_{r}((1+h \beta) /(1+h \alpha))\right\}, \max \left\{k_{r}((1-h \beta) /(1-h \alpha)), k_{r}((1+\right.$ $h \beta) /(1+h \alpha))\}]$.

Proof: Straightforward from Proposition 3.

Corollary 2: The phase of the internal model, $\angle K\left(e^{j \omega}\right)$, in the frequency range $[\varpi, \pi]$, for $k_{r}>0,|\alpha| \leq 1,|\beta| \leq 1, H(z)$ stable and $\left|H\left(e^{j \omega}\right)\right|<h, \forall \omega \in[\varpi, \pi]$ is in the interval $\pm \arctan (h \mid \alpha-$ $\left.\beta \mid / \sqrt{\left(-1+\beta^{2} h^{2}\right)\left(\alpha^{2} h^{2}-1\right)}\right)$ which is always contained in the interval $[-2 h, 2 h] \mathrm{rad}$.

Proof: Straightforward from Proposition 3.

In many situations is interesting to know the value of $h$ to ensure that the internal model gain is lower than $\gamma$ in a particular frequency range. This value can be computed from Corollary 1 .

Remark 3: In order to keep the gain of $k_{r}\left(\left(z^{N}-\beta H(z)\right) /\left(z^{N}-\right.\right.$ $\alpha H(z)))$ lower than $\gamma$ in the frequency range $[\varpi, \pi], H(z)$ should satisfy $\left|H\left(e^{j \omega}\right)\right|<\left|\left(k_{r}-\gamma\right) /\left(k_{r} \beta-\alpha \gamma\right)\right|, \omega \in[\varpi, \pi]$ and $k_{r}<\gamma$.

By choosing a proper set of values for $\alpha, \beta$ and $k_{r}$ and using the bounds introduced in Remarks 1, 2, and 3, it is possible to shape the internal model frequency response according to the desired performance [2].

\section{DESIGN PROCEDURE}

It is well known that the closed-loop negative feedback connection of two passive systems generates a stable system [18]. In this sense the proposed repetitive controller $K(z)$ can be connected to any discretetime passive plant, ${ }^{5} G(z)$, giving a stable closed-loop system. Besides this, the proposed controller structure and parameters will ensure the desired tracking or attenuation performance in the closed-loop system. So, if the plant to be controlled $G(z)$ is DTP, then the closed-loop stability is guaranteed by construction. But, unfortunately, most discrete-time plants are not DTP, for this reason an stabilizing controller, $C_{\text {int }}(z)$, is introduced in order to guarantee closed-loop stability. Several approaches can be used in the design of $C_{i n t}(z)$, for those plants which are feedback passivizable [16]. It is possible to construct a new plant, $P$, which is passive so the complete system (see Fig. 1) will be closed-loop stable by construction. In order to extend the class of plants where the proposed repetitive controller can be applied, the concept of finite-frequency positive real (FFPR) systems [11] is recalled.

Definition 4: A transfer function $G(z)$ is called Finite-Frequency Discrete-Time Positive Real (FFDTPR) with bandwidth $\varpi$ if it satisfies the conditions in Definition 3 with $|\omega|<\pi$ replaced by $|\omega|<\varpi<\pi$.

If a closed-loop system obtained by connecting two FFDTPR systems with bandwidth $\varpi$ or greater, is stable, it will also be FFDTPR with at least the same bandwidth. The stability of the closed-loop system can be checked by applying the Nyquist Criterion. The Nyquist plot of the open-loop system will lie in the frequency range $[0, \varpi]$ on the 4th and 3rd quadrants, to guarantee closed-loop stability it is only necessary to have gain less than one in the complementary frequency range, $[\varpi, \pi]$.

This reasoning can be summarized in the following proposition.

\footnotetext{
${ }^{5}$ Note that may be any linear or nonlinear discrete-time passive plant.
}

Proposition 4: The closed-loop system obtained when two FFDTPR systems, $K(z)$ and $P(z)$, with bandwidth $\varpi$ are connected in feedback loop is stable if

$$
\left|P\left(e^{j \omega}\right)\right|\left|K\left(e^{j \omega}\right)\right|<1, \quad \forall \omega \in[\varpi, \pi] .
$$

Proof: Straightforward from previous comments.

Remark 4: If one of the systems connected in feedback is DTPR and the other is FFDTPR with bandwidth $\varpi$ the previous results also apply.

If the plant to be controlled $G(z)$ is not DTPR and it is not feedback passivizable then the following design procedure is proposed (see Fig. 1):

1) Determine the system bandwidth, $(\varpi)$, in which the plant is PR. If this bandwidth is not large enough for the desired performance, then $G(z)$ can be combined in feedback connection with an stabilizing controller $C_{\text {int }}(z)$ to increase the original plant bandwidth. This procedure will define a new bandwidth $\varpi$ for the modified system $P(z)$. Note that it is necessary that $P(z)$ has low gain (lower than one) in the complementary range of frequencies where it is Positive Real [11].

The design of $C_{\text {int }}(z)$ can be addressed using different techniques such that $C_{i n t}\left(e^{j \omega}\right) G\left(e^{j \omega}\right) /\left(1+C_{i n t}\left(e^{j \omega}\right) G\left(e^{j \omega}\right)\right)$ is FFDTPR with bandwith $\varpi$ while minimizing $\mid C_{i n t}\left(e^{j \omega}\right) G\left(e^{j \omega}\right) /(1+$ $\left.C_{\text {int }}\left(e^{j \omega}\right) G\left(e^{j \omega}\right)\right) \mid$ for $\omega>\varpi$. Some commonly used techniques include phase cancellation [17] and generalized KYP techniques [11].

2) Choose $N, \alpha$ and $\beta$ of the internal model $K(z)=k_{r}\left(\left(z^{N}-\right.\right.$ $\left.\beta H(z)) /\left(z^{N}-\alpha H(z)\right)\right)$ to shape the frequency response according specifications.

3) Calculate $\gamma=\inf _{\omega \in[\varpi, \pi]}\left(1 /\left|P\left(e^{j \omega}\right)\right|\right)$. The value of $k_{r} \in(0, \gamma)$ makes, together with the condition in the next step, the closed-loop system stable and it must be chosen using a trade-off between robustness and time response [8].

4) Finally, to ensure the closed-loop stability, it is necessary to find a lowpass filter, $H(z)$, such that

$$
\left|k_{r} \frac{e^{-j \omega N}-\beta H\left(e^{-j \omega}\right)}{e^{-j \omega N}-\alpha H\left(e^{-j \omega}\right)}\right|<\gamma, \quad \forall \omega \in[\varpi, \pi]
$$

and, by applying Remark 3, this condition can be transformed to the following specification for $H(z)$ :

$$
\left|H\left(e^{j \omega}\right)\right|<\left|\frac{k_{r}-\gamma}{k_{r} \beta-\alpha \gamma}\right|, \quad \omega \in[\varpi, \pi] .
$$

\section{NUMERICAL EXAMPLES}

\section{A. Example I}

This example shows how the proposed internal model can be used with a DTP plant. In this case it can be used without a stabilizing controller, so the complete controller would be simpler than the one obtained with a conventional repetitive controller. It is worth to note that taking advantage of the proposed internal model passivity, several internal models can be combined in a very straightforward manner to obtain good performances with multiperiodic references or disturbances.

In this example, we design a repetitive controller to force the DTP plant, $G(z)=((z-0.6)(z-0.2)) /((z-0.9)(z-0.3))$, to track the signal, $r(k)=2 \sin \left(\left(2 \pi / N_{2}\right) k\right)+0.7 \sin \left(\left(2 \pi / N_{1}\right) k\right)$ with $N_{1}=77$ and $N_{2}=50$. As this signal is composed by two sinusoidal signals with non harmonic frequencies, the multi-periodic internal model is

$$
K(z)=k_{r}\left(\frac{z^{50}+0.6}{z^{50}+1}+\frac{z^{77}+0.6}{z^{77}+1}\right)
$$




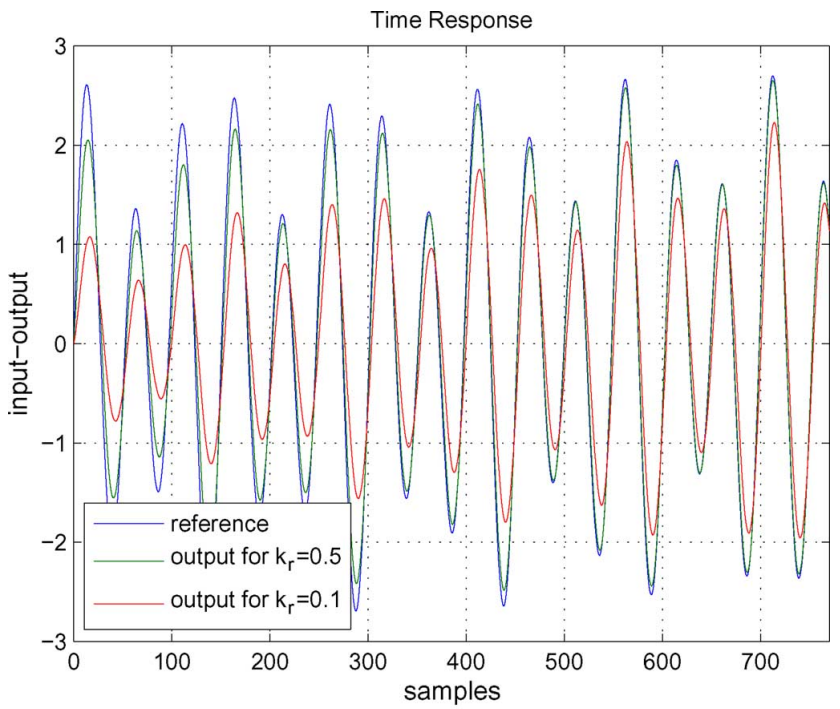

Fig. 2. Closed-loop time response: reference, output with $k_{r}=0.5$ and output with $k_{r}=0.1$.
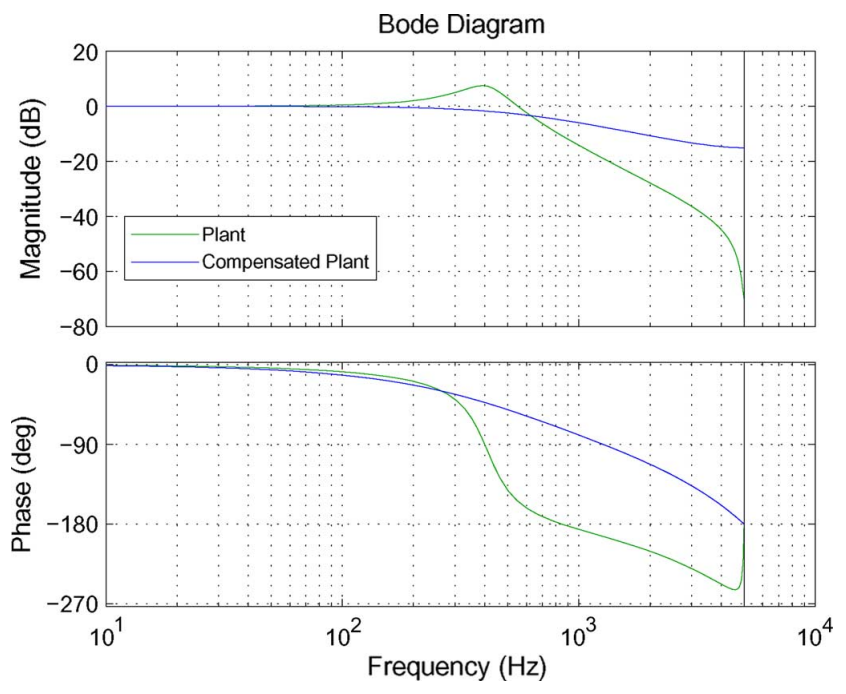

Fig. 3. Bode diagram of the plant $(G)$ and the compensated plant $(P)$.

with a term for each frequency to be tracked. As stated in Remark 2, in this case $K(z)$ will be DTP because it is the addition of two DTP transfer functions. The design with this combination would not be so straightforward with the conventional repetitive internal models. It is important to note that, as both the plant and the controller are DTP, there is no need of an stabilizing controller which is also an important difference with respect to the conventional repetitive control.

Fig. 2 shows the system behavior for two values of $k_{r}$. As expected, in both cases the output follows the reference signal in steady state. Note that higher is $k_{r}$ smaller is the settling time. The repetitive controller does not use the plant model to guarantee closed-loop stability because the plant is DTP and, clearly, this strategy is an improvement in comparison to the conventional repetitive control designs.

\section{B. Example II}

The purpose of this example is to illustrate the design procedure proposed in Section III for FFDTPR plants.

The control objective is to force the plant $G_{p}(z)=(0.03196 z+$ $0.03079) /\left(z^{2}-1.832 z+0.8948\right)$ to track a $F_{p}=50 \mathrm{~Hz}$ sinusoidal signal. This plant corresponds to the discrete-time model of a pulse-width modulated (PWM) dc-ac converter with sampling time

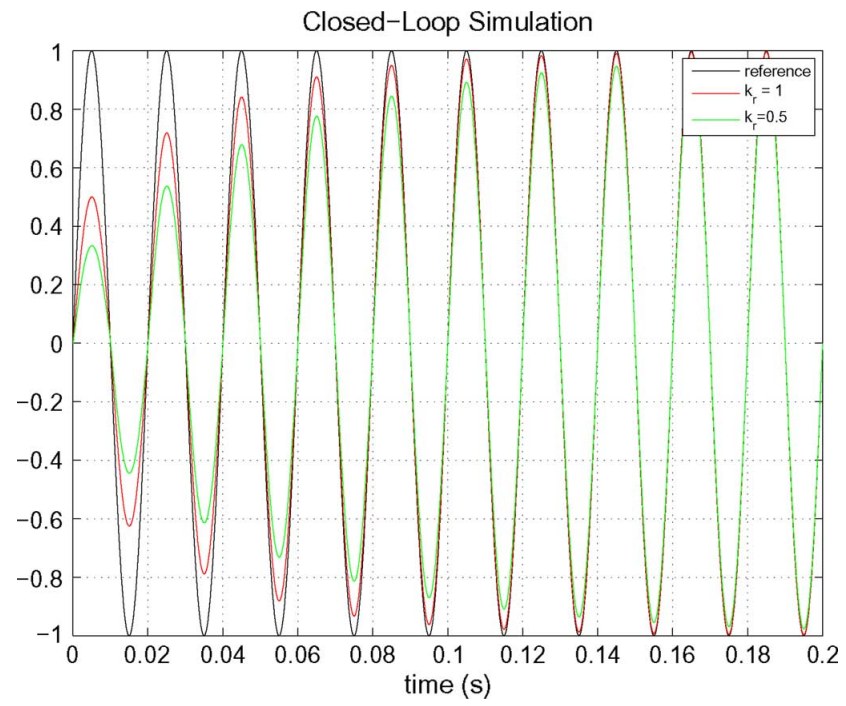

Fig. 4. Closed-loop time response: reference, output with $k_{r}=1$ and output with $k_{r}=0.5$.

$T_{s}=1 / F_{s}=10^{-4} \mathrm{~s}[20]$ and the sinusoidal signal to be tracked is the reference ac voltage of the closed-loop system. The design procedure for this example is as follows:

- Step 1: As shown in Fig. 3, this plant is FFDTPR with bandwidth 6 $\varpi=0.2507 \mathrm{rad}(399 \mathrm{~Hz})$ and $\gamma=\inf _{\omega \in[\varpi, \pi]}\left(1 / P\left(e^{j \omega}\right)\right)=$ 0.43 . Unfortunately, the bandwidth in which this plant is $P R$ is very small. So, in order to allow the internal model to introduce high gain in a broader bandwidth it is needed to increase the bandwidth in which the system is PR. A stabilizing controller, $C_{\text {int }}$, which enlarges PR frequencies range of $P$, is designed following the phase cancellation [17] approach.

Then, the complete plant, $P$, equals to $0.3 /(z-0.7)$ which is FFDTPR with bandwidth $\varpi=0.7956 \mathrm{rad}$ (1266.3 Hz) (Fig. 3). It is important to note that the stabilizing controller, $C_{i n t}$, can be designed using any control methodology and that is a clear improvement when compared to the conventional repetitive control design by a plant inversion method.

- Step 2: In this step the parameters $N, \alpha$ and $\beta$ of the internal model, $K(z)$, are selected. As the frequency of the signal to be tracked is $F_{p}$ and assuming that the possible disturbances of the system are odd-harmonic periodic signals, $N=F_{s} / 2 F_{p}=100$ and $\alpha=-1$ [6]. Furthermore, as a very selective (in frequency) system is desired $\beta=-0.5$. For this particular example, the openloop transfer function has high gain only where it is necessary and, then, the behaviour of the closed-loop system is better in the presence of the measurement noise. This additional degree of freedom is an improvement with respect to the conventional repetitive controllers which do not allow to shape the open-loop frequency response.

- Step 3: Once $P$ is designed, and $N, \alpha$ and $\beta$ are fixed, $\gamma$ is obtained as $\gamma=2.381$. Then, $k_{r}$ can be chosen in the interval $(0$, 2.381).

- Step 4: Selecting $k_{r}=1, H(z)$ is designed according to (8). This condition states that $H(z)$ must have a maximum gain of $\mid\left(k_{r}-\right.$ $\gamma) /\left(k_{r} \beta-\alpha \gamma\right) \mid=0.7342$ in the frequency range $[0.7956, \pi] \mathrm{rad}$ $([1266.3,5000] \mathrm{Hz})$. To obtain this attenuation, a third-order nullphase low-pass FIR filter can be designed accordingly. ${ }^{7}$ With this filter, the order of the controller $K(z)$ is 103 .

${ }^{6}$ The frequency range is $\omega \in[0, \pi]$ with $\pi$ corresponding to half the sampling frequency $F_{s}=1 / T_{s}$.

${ }^{7}$ In this procedure any standard filter design procedure can be used. 
Fig. 4 shows the simulation results obtained when a $50 \mathrm{~Hz}$ sinusoidal signal is used as a reference for two values of $k_{r}$. The results show that, as expected, a lower value of $k_{r}$ gives a slower closed-loop time response. Furthermore, in both cases the output tracks the reference almost perfectly in steady state and there is no delay in the beginning of the time response. This last characteristic is different in the conventional repetitive control systems where the high relative degree of the controller causes a significant delay in the response.

\section{CONCLUSION}

This work has proposed an internal model for repetitive control design. This internal model is a Discrete-Time Passive, or equivalently, Discrete-Time Positive Real. According to this, the proposed internal model can be connected in feedback form with a generic DTP system with the closed-loop stability being ensured.

Taking advantage of the passivity structure, a design procedure for FFDTPR systems has been proposed and analyzed. The design procedure allows to set the desired gain margin through the value of parameter $k_{r}$ and filter $H(z)$. Furthermore, the proposed internal model allows to shape the open-loop frequency response in a more accurate way because it adds an additional degree of freedom to its structure.

Additionally, this passivity structure of the internal model establishes a simple way to deal with multiperiodic references and disturbances.

\section{REFERENCES}

[1] K. Chew and M. Tomizuka, "Digital control of repetitive errors in disk drive systems," IEEE Control Syst. Mag., vol. 10, no. 1, pp. 16-20, Jan. 1990.

[2] R. Costa-Castelló and R. Griñó, "A repetitive controller for discretetime passive systems," Automatica, vol. 42, no. 9, pp. 1605-1610, 2006.

[3] H. Dang and D. Owens, "Mimo multi-periodic repetitive control system: Universal adaptive control schemes," Int. J. Adaptive Control Signal Processing, vol. 20, pp. 409-429, 2006.

[4] B. Francis and W. Wonham, "Internal model principle in control theory," Automatica, vol. 12, pp. 457-465, 1976.

[5] G. C. Goodwin and K. S. Sin, Adaptive Filtering Prediction and Control, ser. Information and Systems Science, T. Kailath, Ed. Englewood Cliffs, NJ: Prentice Hall, 1984.

[6] R. Griñó and R. Costa-Castelló, "Digital repetitive plug-in controller for odd-harmonic periodic references and disturbances," Automatica, vol. 41, no. 1, pp. 153-157, Jan. 2005.

[7] S. Hara, Y. Yamamoto, T. Omata, and M. Nakano, "Repetitive control system: A new type servo system for periodic exogenous signals," IEEE Trans. Automat. Control, vol. AC-33, no. 7, pp. 659-668, Jul. 1988.

[8] G. Hillerström and R. Lee, Trade-Offs in Repetitive Control University of Cambridge, Cambridge, U.K., Tech. Rep. CUED/F-INFENG/TR 294, Jun. 1997.

[9] L. Hitz and B. D. O. Anderson, "Discrete positive-real functions and their applications to system stability," Proc. IEEE, vol. 116, no. 1, pp. 153-155, 1969.

[10] T. Inoue, M. Nakano, T. Kubo, S. Matsumoto, and H. Baba, "High accuracy control of a proton synchroton magnet power supply," in Proc. 8th IFAC World Congress, 1981, pp. 216-220.

[11] T. Iwasaki and S. Hara, "Generalized KYP lemma: Unified frequency domain inequalities with design applications," IEEE Trans. Automat. Control, vol. 50, no. 1, pp. 41-59, Jan. 2005.

[12] T. Iwasaki, S. Hara, and H. Yamauchi, "Dynamical system design from a control perspective: Finite frequency positive-realness approach," IEEE Trans. Automat. Control, vol. 48, no. 8, pp. 1337-1354, Aug. 2003.

[13] W. Lin, "Synthesis of Discrete-Time Nonlinear Systems," Ph.D. dissertation, Washington University, St. Louis, MO, 1993.

[14] D. H. Owens, L. M. Li, and S. P. Banks, "Multi-periodic repetitive control system: A lyapunov stability analysis for mimo systems," Int. J. Control, vol. 20, no. 5, pp. 504-515, Mar. 2004.
[15] D. H. Owens, M. Tomas-Rodriguez, J. Hatönen, and L. Li, "Discrete time linear optimal multi-periodic repetitive control: A benchmark tracking solution," Int. J. Control, vol. 79, no. 9, pp. 991-1001, Sep. 2006.

[16] Z. Tan, Y. Soh, and L. Xie, "Dissipative control for linear discrete-time systems," Automatica, vol. 35, no. 9, pp. 1557-1564, Sep. 1999.

[17] M. Tomizuka, "Zero-phase error tracking algorithm for digital control," J. Dyn. Syst., Meas. Control, vol. 109, pp. 65-68, Mar. 1987.

[18] A. van der Schaft, L2-Gain and Passivity Techniques in Nonlinear Control, ser. Springer Communications and Control Engineering, 2nd ed. Berlin, Germany: Springer-Verlag, 2000, vol. 218.

[19] G. Weiss and M. Häfele, "Repetitive control of mimo systems using $H^{\infty}$ design," Automatica, vol. 35, pp. 1185-1199, 1999.

[20] K. Zhou, D. W. Wang, and K. S. Low, "Periodic errors elimination in CVCF PWM DC/AC converter systems: Repetitive control approach," Proc. Inst. Elect. Eng., vol. 147, no. 6, pp. 694-700, Nov. 2000.

\section{Entropy Optimization Filtering for Fault Isolation of Nonlinear Non-Gaussian Stochastic Systems}

Lei Guo, Liping Yin, Hong Wang, and Tianyou Chai

\begin{abstract}
In this paper, the fault isolation (FI) problem is investigated for nonlinear non-Gaussian systems with multiple faults(or abrupt changes of system parameters) in the presence of noises. By constructing a filter to estimate the states, the FI problem can be reduced to an entropy optimization problem subjected to the non-Gaussian estimation error systems. The design objective for the FI purpose is that the entropy of the estimation error is maximized in the presence of diagnosed fault and is minimized in the presence of the nuisance faults or noises. It is shown that the error dynamics is represented by a nonlinear non-Gaussian stochastic system, for which new relationships are applied to formulate the probability density functions (PDFs) of the stochastic error in terms of the PDFs of the noises and the faults. The Renyi's entropy has been used to simplify the computations in the filtering for the recursive design algorithms. It is noted that the output can be supposed to be immeasurable (but with known stochastic distributions), which is different from the existing results where the output is always measurable for feedback. Finally, simulations are given to demonstrate the effectiveness of the proposed data-driven FI filtering algorithms.
\end{abstract}

Index Terms-Entropy optimization, fault isolation, non-Gaussian systems, non-linear filtering, optimal control.

\section{INTRODUCTION}

Fault detection and isolation (FDI) for stochastic systems has drawn a considerable attention in the past decades, where many effective methodologies have been developed as seen from the survey works

Manuscript received September 19, 2007; revised June 14, 2008. Current version published April 08, 2009. This work was supported by the National Natural Science Foundation of China under Grants 60474050 and 60828007 , as well as the 973 and 863 programme of China. Recommended by Associate Editor V. Krishnamurthy.

L. Guo is with the School of Instrument Science and Opto-Electronics Engineering, Beihang University, Beijing 100191, Chian (e-mail:lguo@buaa.edu. $\mathrm{cn})$.

L. P. Yin is with the Research Institute of Automation, Southeast University, Nanjing 210096, China (e-mail: yinlipingqufu@163.com).

H. Wang is with the Control Systems Center, the University of Manchester, Manchester M60 1QD, U.K. (e-mail: hong.wang@manchester.ac.uk).

T. Y. Chai is with the Research Center of Automation, Northeastern University, Shenyang 110004, China (e-mail: ty-chai@mail.edu.cn).

Digital Object Identifier 10.1109/TAC.2008.2009599 